\title{
Compound Ranking Based on a New Mathematical Measure of Effectiveness Using Time Course Data from Cell-Based Assays
}

\author{
Francisco J. Diaz ${ }^{*}, 1$, Peter R. McDonald ${ }^{2}$, Anuradha Roy², Byron Taylor ${ }^{2}$, Ashleigh Price ${ }^{2}$, \\ Jessica Hall ${ }^{3}$, Brian S.J. Blagg ${ }^{3}$, and Rathnam Chaguturu ${ }^{4}$ \\ ${ }^{1}$ Department of Biostatistics, The University of Kansas Medical Center, Kansas City, KS, USA \\ 2University of Kansas High, Throughput Screening Laboratory, Lawrence, KS, USA \\ ${ }^{3}$ Department of Medicinal Chemistry, University of Kansas, Lawrence, KS, USA \\ ${ }^{4}$ Center for Advanced Drug Research, SRI International, Harrisonburg, VA, USA
}

\begin{abstract}
The half maximal inhibitory concentration $\left(\mathrm{IC}_{50}\right)$ has several limitations that make it unsuitable for examining a large number of compounds in cytotoxicity studies, particularly when multiple exposure periods are tested. This article proposes a new approach to measure drug effectiveness, which allows ranking compounds according to their toxic effects on live cells. This effectiveness measure, which combines all exposure times tested, compares the growth rates of a particular cell line in the presence of the compound with its growth rate in the presence of DMSO alone. Our approach allows measuring a wider spectrum of toxicity than the $\mathrm{IC}_{50}$ approach, and allows automatic analyses of a large number of compounds. It can be easily implemented in linear regression software, provides a comparable measure of effectiveness for each investigated compound (both toxic and non-toxic), and allows statistically testing the null hypothesis that a compound is non-toxic versus the alternative that it is toxic. Importantly, our approach allows defining an automated decision rule for deciding whether a compound is significantly toxic. As an illustration, we describe the results of a cell-based study of the cytotoxicity of 24 analogs of novobiocin, a C-terminal inhibitor of heat shock protein 90 (Hsp90); the compounds were ranked in order of cytotoxicity to a panel of 18 cancer cell lines and 1 normal cell line. Our approach may also be a good alternative to computing the half maximal effective concentration $\left(\mathrm{EC}_{50}\right)$ in studies searching for compounds that promote cell growth.
\end{abstract}

\section{Keywords}

Anticancer drugs; cancer cell lines; cytotoxicity; drug effectiveness; EC50; exponential growth model; high throughput screening; hit selection; $\mathrm{IC}_{50}$

\section{INTRODUCTION}

The half maximal inhibitory concentration $\left(\mathrm{IC}_{50}\right)$ is used to assess and compare the effectiveness of inhibitory compounds. In particular, it has been regularly used to investigate the toxicity of compounds in cell-based assays, e.g. assays searching for novel anticancer

\footnotetext{
(C) 2013 Bentham Science Publishers

*Address correspondence to this author at the Department of Biostatistics, University of Kansas Medical Center, Mail Stop 1026, 3901 Rainbow Blvd., Kansas City, KS 66160, USA; Tel: (913) 945-7006; Fax: (913) 588-0252; fdiaz@ kumc.edu.

CONFLICT OF INTEREST

The authors confirm that this article content has no conflict of interest.
} 
drugs. However, the mechanism of action of a toxin is not analogous to the simple receptorligand-interaction mechanism that usually justifies using the $\mathrm{IC}_{50}$. In fact, the $\mathrm{IC}_{50}$ has some limitations that make it unsuitable for the simultaneous examination of a large number of compounds in high throughput screening (HTS) settings. These limitations stem from the inappropriate application of $\mathrm{IC}_{50}$ to assays and situations that were not originally intended for the $\mathrm{IC}_{50}$ calculation. The limitations of the $\mathrm{IC}_{50}$ in HTS situations are: (1) it requires a well shaped dose response curve, (2) it requires a wide range of concentrations of antagonist tested, (3) $\mathrm{IC}_{50}$ measurements cannot be directly compared across assays with different time frames of antagonist exposure, and (4) $\mathrm{IC}_{50}$ measurements cannot be used to statistically test the null hypothesis that a compound is not toxic. The purpose of this article is to propose a new measure of compound effectiveness that overcomes these limitations when two or more exposure times are examined for each compound in a cell-based HTS setting.

Next we discuss the practical impact of the above limitations on HTS. Since the $\mathrm{IC}_{50}$ can be reliably computed only when the cell-growth-inhibition versus compound concentration curve has reached a plateau, its computation requires a previous visual inspection of this curve for each compound; as a consequence of this, an automatic computation of the $\mathrm{IC}_{50}$ is usually difficult and, especially when dealing with a large number of compounds, human errors are bound to happen and the comparison of compounds is very time-consuming. Also, with the low concentrations that are usually tested in HTS, it is common that many curves do not reach a clear plateau. In the absence of a sigmoidal relationship of compound concentration to response, no reliable $\mathrm{IC}_{50}$ measurement can be obtained. Consequently, the $\mathrm{IC}_{50}$ concept is limited in its ability to provide tools for comparing compounds tested in HTS on a quantitative basis.

The $\mathrm{IC}_{50}$ is time-dependent, that is, its value and the possibility of computing it depend on the duration of the exposure of the cells to the compound. This is not a shortcoming by itself if all investigated compounds are tested on the same exposure time and an $\mathrm{IC}_{50}$ for all compounds is available. However, this does create complications for the statistical analysis of data from screens that test the toxicity of the same compounds for two or more exposure times. In particular, it is unclear how to compute a unified measure of compound effectiveness that combines the available $\mathrm{IC}_{50}$ 's from different exposure periods. A commonly adopted practical solution is to use only one exposure time in compound comparisons. Unfortunately, ignoring the information from other available exposure times is clearly an insufficient evaluation of efficacy and excludes the possibility of computing a more precise measure of effectiveness that combines all the available information.

Numerous examples from literature provide evidence that the exposure time of a compound has a direct effect on $\mathrm{IC}_{50}$ measurements [1-2]. The ability to analyze the effects exerted by a compound at a given concentration over a series of time points can provide invaluable information for the optimal concentration for efficacy and duration of action. Obtaining this information as early as possible during the drug discovery and development process is economically ideal and affords preliminary insight into the pharmacokinetic and pharmacodynamic properties of a given compound.

In cell-based HTS settings, there is a need for statistical methods that allow testing the null hypothesis that a compound is not toxic versus the alternative hypothesis that it is. A limitation of the $\mathrm{IC}_{50}$ approach to measuring cytotoxicity is that it cannot be used to implement methods of testing this null hypothesis. The reason is that the $\mathrm{IC}_{50}$ concept is undefined for non-toxic compounds, which implies that any $\mathrm{IC}_{50}$ measurement is computed under the assumption that the compound is toxic. Consequently, an acceptance region for the null hypothesis cannot be defined by using a test statistic based on $\mathrm{IC}_{50}$ measurements, and 
automated decision rules that allow deciding whether a compound is significantly toxic cannot be designed by using $\mathrm{IC}_{50}$ measurements.

Another method that is used to measure drug effectiveness is the area under the curve. This method quantifies the area under the dose-response curve of compound effectiveness, e.g. inhibitor versus compound concentration. Although this area is always computable and can be computed automatically (at least when using a trapezoidal rule), even when no plateau on the curve is observed, the fact that this measure is also time-dependent causes analogous difficulties to those described above for computing $\mathrm{IC}_{50}$ values when two or more exposure times are examined.

The approach proposed in this article possesses none of the disadvantages mentioned above for the $\mathrm{IC}_{50}$ or the area under the curve. In particular, our approach provides a measure of effectiveness that combines all exposure times tested, allowing the automatic computation of the effectiveness of a large number of compounds. This new method can be easily implemented in linear regression software, provides a comparable measure of effectiveness for each investigated compound, and allows testing the null hypothesis that a compound is non-toxic versus the alternative that it is toxic. We describe herein empirical evidence that suggests that our approach provides measures of effectiveness that highly correlate with $\mathrm{IC}_{50}$ measurements when the latter are available. Thus, our approach seems to retain the best features of the $\mathrm{IC}_{50}$ approach while overcoming some of its observed disadvantages in HTS analyses involving multiple times. As described in the Discussion Section, our approach can also be a good alternative to the half maximal effective concentration $\left(\mathrm{EC}_{50}\right)$ in HTS investigations searching for compounds that promote cell growth; this additional application only requires a slight modification to the interpretation of our measure of effectiveness.

To illustrate the proposed methodology, we describe the results of an HTS study of the cytotoxicity of 24 rationally-designed analogs of novobiocin, a C-terminal inhibitor of heat shock protein 90 (Hsp90). The goal of this cell-based assay was to rank each of the 24 compounds in order of cytotoxicity to a panel of 18 cancer cell lines and 1 normal cell line.

Chaperone heat shock proteins play crucial roles in protein folding, assembly of multiprotein complexes, transport of proteins into correct subcellular compartments, cell-cycle control and signaling, and the protection of cells against stress/apoptosis [3-4]. Hsp90 is a potential therapeutic target for anticancer therapy as it regulates the activity of over 200 client proteins, impacting numerous cellular pathways in both normal and cancer cells [4-7]. Drugs that specifically block Hsp90 function contain antitumor activity, and many Hsp90 client proteins are kinases involved in cell proliferation, making Hsp90 an ideal target for anticancer therapeutics. Inhibition of Hsp90 leads to depletion of client proteins involved in the major hallmarks of cancer, including evading apoptosis and detection by the immune system, angiogenesis, invasion and metastasis [7]. In addition, increased Hsp90 expression is observed in cancerous cells in attempts to maintain homeostasis in the hostile environment caused by neoplastic transformation. This upregulation provides a degree of selectivity over non-cancerous cells and could reduce toxicity by selectively inhibiting the progression of cancer-associated Hsp90. The power of specific Hsp90 inhibitors rests with their ability to simultaneously disrupt multiple oncogenic signaling pathways [5, 8]. Clinical trials with current Hsp90 inhibitors have been hampered by limited efficacy and moderate systemic toxicity unrelated to Hsp90 inhibition. The analogs of novobiocin that are examined in this article were synthesized with the purpose of developing more potent and specific inhibitors of Hsp90 for use in developing anticancer chemotherapeutics [9-13]. 


\section{MATERIALS AND METHODS}

The investigated compounds are listed in the first column of Table 2. Twenty-four of these compounds (named with a KU prefix) were synthesized at the University of Kansas; 17AAG and novobiocin were also examined as a standard for Hsp90 inhibition. The cytotoxic effects of these compounds on 19 cell lines were studied. The cell lines are described in Table 1. A schematic diagram of our overall approach is summarized in Fig. (1).

\section{Cell Viability Assays}

Cell viability after exposure to a specific concentration of a compound was assessed using the CellTiter-Glo homogenous luminescent cell viability assay (Promega Co., Madison, WI). Cells exposed to the compound were incubated at $37^{\circ} \mathrm{C}$ and $5 \% \mathrm{CO} 2$ in a $95 \%$ humidified incubator. Four different exposure durations were implemented (4, 24, 48, or 72 hours). After incubation, the cells were removed from the incubator and left at room temperature for 20 minutes to equilibrate the plate and its contents to room temperature. CellTiter-Glo reagent was added to the cells, at $10 \mu \mathrm{L}$ per well, and plates were shaken for two minutes at room temperature and $1600 \mathrm{rpm}(250 \mathrm{rcf})$ speed. Fifteen minutes later, plate luminescence was read on the PerkinElmer Envision to quantitate cell viability. The luminescence values used for data analysis reflected the number of viable cells. Viability was detected by quantitation of the ATP present, which confirms the presence of metabolically active cells. The CellTiter-Glo reagent acts by lysing cells and generating a luminescent signal proportional to the amount of ATP present, and therefore proportional to the number of cells present. The luciferase reaction was based on mono-oxygenation of luciferin, catalyzed by luciferase in the presence of $\mathrm{Mg} 2+$, ATP and molecular oxygen.

\section{Compound Plate Preparation}

Compounds were received at $10 \mathrm{mM}$ in $50 \mu \mathrm{L}$ of $100 \%$ dimethyl sulfoxide (DMSO). They were diluted to $6 \mathrm{mM}$ (100\% DMSO) and placed in columns 3 and 13 of 384-well polypropylene plates ( $83.3 \mu \mathrm{L}$ of each $6 \mathrm{mM}$ compound). Serial dilutions were performed (in 100\% DMSO) for compounds in column 3 across to column 12, and compounds in column 13 were diluted across to column 22 . The serial dilutions were performed by transferring $33.3 \mu \mathrm{L}$ from each well to receiving well containing $50 \mu \mathrm{L}$ DMSO. After mixing, $33.3 \mu \mathrm{L}$ were transferred to the next well containing $50 \mu \mathrm{L}$ DMSO and so on. The final volume in each well was $50 \mu \mathrm{L}$. For each of the investigated compounds, 10 different compound concentrations were tested on the cells: $30.0 \mu \mathrm{M}, 18.0 \mu \mathrm{M}, 10.8 \mu \mathrm{M}, 6.5 \mu \mathrm{M}, 3.9$ $\mu \mathrm{M}, 2.3 \mu \mathrm{M}, 1.4 \mu \mathrm{M}, 0.840 \mu \mathrm{M}, 0.504 \mu \mathrm{M}$, and $0.302 \mu \mathrm{M}$. For each of the 19 cell lines, cell growth curves were used to determine how many initial cells to seed per well of each plate.

A total of 4 plates were used for each cell line. Each plate corresponded to 1 of the 4 compound-exposure durations (4, 24, 48 or 72 hours), allowed testing for 10 different concentrations of each of the investigated compounds and also allowed testing of the DMSO vehicle alone.

\section{Statistical Model of Luminescence Measures}

For a particular compound and cell line, the following notation will be used: $T=$ time at which luminescence is measured (in the Hsp90 study, $T=4,24,48$ or 72 hours; initial seeding occurs at $T=0$ ); $N=$ number of cells in a well at a particular time $T ; L=$ measured luminescence when there are $N$ cells in the well; $N_{0}=$ number of cells initially seeded in the well; $L_{0}=$ luminescence corresponding to $N_{0}$ cells (not measured in the Hsp90 study); and $\lambda$ $=$ growth rate per cell of the cell population (dependent on compound dose). 
The exponential cell growth model was used to describe cell proliferation [14]. According to this model, $\frac{d N}{d T}=\lambda N$; thus, $N=N_{0} \exp (\lambda T)$. This is the standard model of population size when conditions are kept constant and the population has sufficient availability of resources such as nutrients and water over the observation time period.

Our approach to measure drug effectiveness assumes that there is a proportional relationship between luminescence and the number of cells in the well. The manufacturer's report for the CellTiter-Glo luminescent assay provides evidence of this proportional relationship [15], and we confirmed this relationship in our lab by testing MCF-7 cells using a range of 4125,000 cells per well. The proportionality assumption states that $L=k N$, where $k$ is a proportionality constant. Thus, by the exponential growth model and this assumption, for a particular compound concentration and cell line,

$$
\frac{L}{k}=\frac{L_{0}}{k} \exp (\lambda T)
$$

This implies that

$$
\log (L)=\log \left(L_{0}\right)+\lambda T,
$$

where $\log (L)$ is the natural $\log$ arithm of $L$. It is important to note that $\log \left(L_{0}\right)$ is unknown but constant across all assays corresponding to a particular cell line, because the initial number of cells, $N_{0}$ was the same for all compounds and concentrations tested. (Since luminescence was proportional to the amount of viable cells in the well, we did not expect that $\log \left(L_{0}\right)$ was affected by sample concentration). For a particular compound, let $C_{i}, i=1, \ldots, n$, denote the $n$ different concentrations tested on the cell line (in the Hsp90 study, $n=10$ ). It is assumed that these $n$ concentrations were used for all investigated compounds. Each of these concentrations contributes to an equation like equation (1), where $\lambda$ depends on the particular compound concentration tested.

Thus, for a particular cell line and compound $d$, the luminescence measures can be modeled with the following set of $n+1$ equations:

$$
\begin{gathered}
\log (L)=\log \left(L_{0}\right)+\lambda_{d, i} T+\text { error, } \quad i=1, \ldots, n, \\
\log (L)=\log \left(L_{0}\right)+\lambda_{0} T+\text { error } \quad \text { (DMSO equation), }
\end{gathered}
$$

where $\lambda_{d, i}$ is the growth rate per cell of the investigated cell line in the presence of compound $d$ at concentration $C_{i}, i=1, \ldots, n$; and $\lambda_{0}$ is its growth rate in the presence of only DMSO vehicle (compound concentration $=0$ ).

After combining the above $n+1$ equations, and since $\log \left(L_{0}\right)$ is the same in all $n+1$ equations, we obtain the following linear regression model of an individual luminescence measure $L$ from a particular combination of compound and cell line:

$$
\log (L)=\log \left(L_{0}\right)+\lambda_{0} T X_{0}+\sum_{i=1}^{n} \lambda_{d, i} T X_{i}+\text { error }
$$

where $X_{0}=1$ if the cells in the well were exposed to DMSO alone, and $X_{0}=0$ otherwise; and $X_{i}=1$ if the cells were exposed to compound $d$ at concentration $C_{i}$, and $X_{i}=0$ if the cells were exposed to a compound concentration other than $C_{i}$ or to DMSO alone, $i=1, \ldots, n$. 
Observe that the growth rates $\lambda_{d, i}$ and $\lambda_{0}$ in model (3) are regression coefficients, and $\log \left(L_{0}\right)$ can be estimated as an intercept. To see why equation (3) represents all $n+1$ equations in (2), observe that, for instance, if $L$ is a particular luminescence measure from a well that contained cells exposed to compound concentration $C_{1}$ during $T$ units of time, then $X_{1}=1$ and $X_{i}=0$ for all $i \neq 1$. Entering these numbers into equation (3), we obtain that

$$
\begin{aligned}
\log (L)=\log \left(L_{0}\right)+\left(\lambda_{0}\right)(T)(0)+ & \left(\lambda_{d, 1}\right)(T)(1)+\left(\lambda_{d, 2}\right)(T)(0)+\ldots+\left(\lambda_{d, n}\right)(T)(0)+\text { error } \\
& =\log \left(L_{0}\right)+\lambda_{d, 1} T+\text { error }
\end{aligned}
$$

which is the equation corresponding to concentration $C_{1}$ in (2). Analogous arguments can be used to obtain the other equations in (2) from (3).

Observe that, to be able to fit model (3) with standard linear regression software to data from one particular combination of compound and cell line, the database must initially contain $n$ +3 columns ("variables"), and each row in the database must contain information corresponding to only one luminescence measure, that is, information from only one well. Specifically, one column must contain all the luminescence measures $L$ obtained from the particular combination of compound and cell line; another column must contain the times $T$ at which the corresponding luminescence measures were obtained; and the remaining $n+1$ columns must contain the values of $X_{0}, X_{1}, \ldots, X_{n}$. For a particular row in the database, only one entry of these latter $n+1$ columns should have a value of 1 , and the other entries of the $n$ +1 columns should be 0 , because each well is treated with one and only one compound concentration. Once this database is in place, additional columns $T X_{0}, T X_{1}, \ldots, T X_{n}$, and $\log (L)$ need to be calculated. The columns $T X_{0}, T X_{1}, \ldots, T X_{n}$ are the independent variables of model (3), whereas $\log (L)$ is the dependent variable.

\section{Effectiveness Measure}

The effectiveness of compound $d$ at concentration $C_{i}$ was defined as

$$
E_{d, i}=\left(1-\exp \left(-r\left(\lambda_{0}-\lambda_{d, i}\right)\right)\right) \times 100, \quad i=1, \ldots, n, \quad \text { (4) }
$$

where $r$ is the longest time at which luminescence was measured (in the Hsp90 study, $r=72$ hours). If $r$ is measured in hours, $E_{d, i}$ is interpreted as the proportion of cells killed by compound $d$ at concentration $C_{i}$ after $r$ hours of exposure to the compound. This interpretation is justified by the equation

$$
E_{d, i}=\frac{N_{0} \exp \left(r \lambda_{0}\right)-N_{0} \exp \left(r \lambda_{d, i}\right)}{N_{0} \exp \left(r \lambda_{0}\right)} \times 100
$$

That is, $E_{d, i}$ compares the number of surviving cells after $r$ units of time of uncontrolled growth (i.e. under DMSO alone) with the number of cells that survived after $r$ units of time of compound exposure, regardless of the initial number of cells.

The overall effectiveness $\varepsilon_{d}$ of a compound $d$ was defined as the area under the curve for the plot of $E_{d, i}$ versus $C_{i}$, computed with the trapezoidal rule (Fig. 2); that is:

$$
\varepsilon_{d}=\sum_{i=1}^{n-1} \frac{C_{i+1}-C_{i-1}}{2} E_{d, i}+\frac{C_{n}-C_{n-1}}{2} E_{d, n},
$$


where $\mathrm{C}_{0}=0$. A positive value of $\varepsilon_{d}$ indicates compound toxicity and a negative value of $\varepsilon_{d}$ indicates that the compound favors cell growth.

\section{Model Fit}

For a particular combination of compound and cell line investigated in the Hsp90 study, the values of the covariates $T X_{i}, i=0,1, \ldots, 10$, were computed for each well of the 4 plates. (A well contained cells treated with a particular concentration of compound, or contained cells in DMSO alone; moreover, each well has an associated exposure time $T$ ). These covariates and their corresponding luminescence measures were used to fit the linear regression model (3) using Stata [16].

For each cell line, before fitting the regression models, the adequacy of the exponential growth model was examined by plotting the log of luminescence versus time using all the DMSO-only data from that cell line. This growth model predicts that the plotted points should follow a linear relationship (Fig. 3). Although only 4 time points were available, a linear relationship was observed for 13 of the 19 cell lines. For these cell lines, all available information was used to fit the regression models. For 5 of the 6 cell lines that did not exhibit a linear relationship (HT-1376, Jurkat, MRC-5, NCI-H226 and SK-BR-3), linearity was obtained by excluding the DMSO-only data corresponding to one of the 4 time points. For instance, for MRC-5, a linear relationship was obtained after excluding the 72-hours exposure DMSO data. In order to obtain a reliable estimator of $\lambda_{0}$, which is the reference growth rate, the regression models for these 5 cell lines were fitted by using only the DMSO-only data corresponding to the 3 time points that yielded the linearity, plus all compound-exposure data. One cell line, Coav-3, did not exhibit any obvious linearity in the relationship between the log of luminescence and time, not even after leaving only 3 time points. For Coav-3, however, regression models were fitted using all the available information.

Each combination of compound and cell line provided a total of 96 luminescence measures (10 for compound exposures and 14 for DMSO-only exposures from each of the four different exposure durations). Thus, for the 5 cell lines for which one time point from DMSO data was excluded, the design matrix of the regression model contained a total of 82 rows. For the remaining 14 cell lines, it contained 96 rows.

To test the null hypothesis $H_{0}: \varepsilon_{d} \leq 0$ (the compound is non-toxic) versus the alternative $H_{1}$ : $\varepsilon_{d}>0$ (the compound is toxic), a 1-tailed t-test was used. Since $\varepsilon_{d}$ is a nonlinear contrast of the regression coefficients of model (3), the delta method was used to compute its standard error [17]. Stata's nlcom command was used for these computations. For each cell line, pvalues from t-tests were corrected to account for multiple comparisons. Simes' method was used for these corrections, which controls the false discovery rate [18]; the method started with an uncorrected overall critical p-value of 0.05 .

\section{$\mathrm{IC}_{50}$ Assessments}

For comparison purposes, independent measures of $\mathrm{IC}_{50}$ 's were obtained by using the data corresponding to a 72-hour exposure (Table 3). For reasons similar to those described in the introduction, it was not possible to obtain reliable $\mathrm{IC}_{50}$ measurements for all compounds or all cell lines. Only reliable measurements are reported in Table 3 . The chemist who obtained $\mathrm{IC}_{50}$ measurements had substantial prior experience computing $\mathrm{IC}_{50}$ measurements and was not aware of the model or its results at the time of $\mathrm{IC}_{50}$ computations. Also, model development and effectiveness computations were done without knowledge of results from $\mathrm{IC}_{50}$ computations. For each cell line that provided $\mathrm{IC}_{50}$ measurements, a Spearman's correlation across compounds between available $\mathrm{IC}_{50}$ 's and their corresponding $\varepsilon_{d}$ 's was 
computed (last row of Table 3). Similarly, for each compound that provided $\mathrm{IC}_{50}$ measurements, a Spearman's correlation across cell lines was computed (last column of Table 3). Correlation coefficients were reported only if there were 3 or more pairs $\left(\mathrm{IC}_{50}\right.$, overall effectiveness) available.

\section{Assessment of the Goodness-of-the-Fit of the Linear Model}

For each combination of compound and cell line, the goodness-of-the-fit of linear regression model (3) was assessed through the correlation coefficient between the predicted and observed log-transformed luminescence measures; this correlation is given by the square root of the usual coefficient of determination $\mathrm{R}^{2}$. For each combination of compound and cell line, a correlation coefficient was computed (Table 4).

\section{RESULTS}

For each cell line, Table 2 shows the values of overall effectiveness $\varepsilon_{d}$ for the investigated compounds. Overall effectivenesses that were significantly greater than 0 , which indicates a statistically significant toxicity, are highlighted. For instance, the overall effectiveness of compound KU-174 for killing A549 cells was $\varepsilon_{d}=1921.3\left(13^{\text {th }}\right.$ column, $2^{\text {nd }}$ row of the table); this effectiveness was significantly greater than 0 . In contrast, the overall effectiveness of compound KU-124 for killing A549 cells was positive but not significantly greater than $0\left(\varepsilon_{d}=376.7\right)$.

The rows in Table 2 are sorted from highest to lowest compound toxicity (up to down). To sort the rows, all $\varepsilon_{d}$ 's for each compound $d$ were added up across columns. Then the rows were sorted from the highest to the lowest value of this sum. Similarly, the columns in Table 2 are sorted from highest to lowest cell line's sensitivity (left to right), by using the sums of $\varepsilon_{d}$ 's across rows (the higher the sum the more sensitive the cell line was to the set of tested compounds).

Table 2 shows that KU-174, 17-AAG and KU-135 were the most toxic compounds, and that Novobiocin was the least toxic. In fact, novobiocin was toxic to only the normal cell line, MRC-5 $\left(\varepsilon_{d}=783.6\right)$.

\section{Comparisons with Available $\mathrm{IC}_{50}$ Measurements}

All correlation coefficients between available $\mathrm{IC}_{50}$ 's and effectiveness measurements $\varepsilon_{d}$ were negative, and most were substantially high in absolute value (Table 3). For instance, for the Jurkat cell line, $\mathrm{r}=-0.82(\mathrm{p}<0.001, \mathrm{~N}=15$; last row of Table 3$)$, and for the KU-135 compound, $r=-0.93(p=0.001, N=8$; last column of Table 3). Eight out of nine within-cell correlations were both $\leq-0.7$ and significantly different from 0 (last row of Table 3 ). Similarly, eight out of 11 within-compound correlations were $\leq-0.7$, although only 5 of these were significant (last column of Table 3). The significant within-compound correlations were those computed with sample sizes $\geq 7$, whereas the non-significant withincompound correlations were computed with sample sizes $<7$; this suggests that the nonsignificant p-values in the last column of Table 3 may be due to a lack of statistical power determined by sample sizes that were not sufficiently large.

The correlations reported in Table 3 suggest that $\varepsilon_{d}$ may convey the same information as the $\mathrm{IC}_{50}$ does when the latter is available. In fact, the negative correlations in Table 3 demonstrate that compounds with experimentally derived low $\mathrm{IC}_{50}$ values (an indication of toxicity at low concentrations) tend to have high $\varepsilon_{d}$ values, which supports our view that $\varepsilon_{d}$ can be interpreted as a measure of compound toxicity. However, the $\mathrm{IC}_{50}$ could not be measured in many instances where the compound was significantly toxic according to the 
overall effectiveness $\varepsilon_{d}$ (Table 3 ), suggesting that focusing on computing only the $\mathrm{IC}_{50}$ in HTS analyses may lead to loss of information.

Table 3 also shows that $\mathrm{IC}_{50}$ 's tended to be available only for compounds that had large values of $\varepsilon_{d}$. In fact, a negative or relatively low effectiveness $\varepsilon_{d}$ was correlated with the inability to measure the $\mathrm{IC}_{50}$ under the tested concentrations. An extreme situation occurred for the MCR-5 cell line, where the $\mathrm{IC}_{50}$ was computable only for the compound with the highest toxicity according to our approach (17-AAG; Table 3). Thus, our approach not only reflects the information that is usually provided by the $\mathrm{IC}_{50}$, but is also able to measure a wider spectrum of toxicity.

\section{GOODNESS OF THE FIT OF THE LINEAR MODEL}

Table 4 describes the correlation coefficients between observed and predicted logtransformed luminescence measures, where the predictions were provided by model (3). Each row in Table 4 shows the minimum, maximum and average correlation of a set of 26 correlations corresponding to the 26 investigated compounds. For most cell lines, model (3) fit remarkably well. In fact, for 13 out of the 19 cell lines, the correlations between observed and predicted log-luminescence measures were of at least 0.80 across the 26 investigated compounds (Table 4). Some of the investigated cell lines (LNCap and HT-29) exhibited an almost perfect model goodness-of-fit for each of the 26 compounds, with an average correlation of 0.99 and minimum correlations not lower than 0.93 .

The lowest (across cell lines) average correlation was 0.60 (Table 4), which still can be considered as indicating a model with reasonably good predictive capabilities, at least from a practical point of view. The poorest model fits were observed from the 26 regressions for the Coav-3 cell line (Table 4), which is not surprising because initial graphical analyses showed that for this cell line there did not seem to be linearity in the relationship between $\log$-transformed luminescence and time under DMSO alone. The minimum and average correlations for the Coav- 3 cell line ( 0.53 and 0.60 , respectively) were the lowest among all investigated cell lines, although compounds producing correlations of up to 0.85 were observed for Coav-3.

The strong evidence of goodness-of-fit in Table 4, and the high correlations between the $\mathrm{IC}_{50}$ and our measure of overall compound effectiveness in Table 3, provide evidence that our linear model has good predictive capabilities and strongly support the usefulness of our model as a tool in HTS studies.

\section{DISCUSSION}

This article describes a statistical methodology for ranking compounds according to their toxic effects on live cells. Our approach is theoretically sound, since it makes use of a well established model in mathematical biology, the exponential growth model [14], and of known quantitative properties of a luminescent assay [15]. Our approach computes a measure of compound effectiveness that essentially compares the growth rates of a particular cell line in the presence of the compound with its growth rate in the presence of only DMSO. We found compelling evidence that the exponential growth model is applicable in an HTS setting, by using luminescence measurements obtained after cell exposure to DMSO alone (Fig. 3). Unfortunately, the adequacy of the exponential growth model could not be directly examined by plotting data from cells exposed to the investigated compounds, because only 4 observations per investigated compound concentration were available. However, we were able to examine the goodness-of-the-fit of a linear model that is implied by the exponential growth model, namely model (3), by examining the correlations between predicted and observed log-transformed luminescences. The linear model fit relatively well 
in most of the investigated cell lines (Table 4). Our computations of overall compound effectiveness relied on this linear model. A similar measure of compound effectiveness to that in formula (4) was successfully used to investigate the effects of mechanical forces on the efficacy of anti-lung cancer drugs [19].

We investigated the performance of our approach by using data from an HTS study of the toxic effects of a family of compounds on 19 cell lines, of which 18 were cancerous. We found that the proposed approach enhanced our ability to measure the toxicity of the investigated compounds, allowing us to measure a wider range of cytotoxicity than the range allowed by the $\mathrm{IC}_{50}$ approach. In particular, our approach seems to be more suitable for comparing compounds with low levels of toxicity since, in this class of compounds, the $\mathrm{IC}_{50}$ approach may not produce any reliable number if low dosages are tested in the experiments.

An advantage of the proposed approach is that it combines all available information durations of exposure to the compound, compound concentrations, and luminescence measurements for cells that were in the presence of the compound versus vehicle DMSOin only one measure of toxicity, $\varepsilon_{d}$. In particular, the use of luminescence measures in the presence of DMSO vehicle alone allows considering $\varepsilon_{d}$. as a standardized measure of toxicity. Another advantage of our approach over the $\mathrm{IC}_{50}$ approach is that a statistical test of the null hypothesis that the compound is not toxic $\left(\varepsilon_{d} \leq 0\right)$ can be performed. In fact, such a test cannot be performed by using $\mathrm{IC}_{50}$ 's because, strictly speaking, the $\mathrm{IC}_{50}$ is defined only for toxic compounds; in other words, any $\mathrm{IC}_{50}$ measure assumes that the compound has some level of toxicity. An $\varepsilon_{d}$ measure, in contrast, does not assume that the investigated compound has any level of toxicity. Finally, our approach can easily be implemented in linear regression software and is more suitable for automation. The latter characteristic is a great advantage, especially in HTS analyses examining thousands of compounds.

We found that our proposed approach produces a measure of effectiveness that highly correlates with $\mathrm{IC}_{50}$ measures in cases in which the latter are obtainable. Remarkably, this correlation was high for the Coav-3 cell line ( $\mathrm{r}=-0.89, \mathrm{p}=0.02, \mathrm{~N}=6$; last row of Table 3 ), which provided DMSO-only data that did not seem to favor the exponential growth model. The correlation was also high for the Jurkat cell line $(r=-0.83, \mathrm{p}=0.001, \mathrm{~N}=15)$, for which we eliminated the DMSO-only data obtained at 24 hours before fitting the regression models. Even more, for the Jurkat cell line the compounds with missing $\mathrm{IC}_{50}$ measurements in Table 3 corresponded to those that had negative or small $\varepsilon_{d}$ values in the corresponding column in Table 2. These high correlations under rather unfavorable or "doubtful" conditions suggest the robustness of our approach.

For 4 of the 5 cell lines for which one time point was excluded from the DMSO-only data in order to achieve visual linearity in the relationship between log-transformed luminescence and time under only DMSO, the average correlation measuring goodness of fit was substantially high (HT-1376, average =0.88; Jurkat, 0.90; SK-BR-3, 0.86; and NCI-H226, 0.93; Table 4). Interestingly, for these cell lines, each regression model was fitted by using 42 luminescence measures under DMSO alone, plus 40 measures under compound concentrations greater than 0 . This means that these latter luminescence measures were quite consistent with the exponential growth model. Moreover, Table 3 shows that, for the HT-1376 and Jurkat cell lines, the correlations between available $\mathrm{IC}_{50}$ measures and overall compound effectivenesses were high $(-0.8$ and -0.83 , respectively). These facts suggest that an initial preparation of DMSO-only data in order to make these data consistent with the assumption of cell exponential growth may produce useful results, and that a seemingly lack of conformity to the exponential growth model may perhaps be owing to some experimental artifacts that may not consistently occur at all exposure durations, and may not be owing to a 
lack of biological conformity of cells to the exponential growth model under HTS conditions.

A limitation of our approach is that it is not applicable when only one exposure duration has been implemented in the HTS experiments. The reason for this is that the luminescence $L_{0}$ corresponding to the initial number of cells $N_{0}$ is not usually available. (This is also the reason why the intercept $\log \left(L_{0}\right)$ in model (3) needs to be estimated). In contrast, when there is only one exposure duration, the area under the inhibition versus concentration curve can always be computed with the trapezoidal rule; the $\mathrm{IC}_{50}$ can or cannot be computed, depending on whether or not a clear plateau in this curve is reached.

Our approach also allows measuring "negative" toxicities (i.e. $\varepsilon_{d}<0$ ), which reflect that a compound favors cell growth. It is likely that $\varepsilon_{d}$ is a better, alternative measure to the $\mathrm{EC}_{50}$, which is typically used in cell-based HTS explorations focused on finding compounds that stimulate cell growth. In fact, $\mathrm{EC}_{50}$ 's essentially have the same limitations as $\mathrm{IC}_{50}$ 's. In those explorations, a low negative value of $\varepsilon_{d}$ can be interpreted as reflecting a high efficacy, and a minor modification of the statistical test described in the Methods Section can easily be carried out in order to test the null hypothesis of lack of compound efficacy $\left(\varepsilon_{d}\right.$ $\geq 0)$ versus the alternative of efficacy $\left(\varepsilon_{d}<0\right)$.

Standard linear regression software usually applies ordinary least squares (OLS) by default in order to estimate the regression coefficients of a linear regression model. In the context of model (3), this estimation procedure will assume that the variance of the error is the same across all different concentrations tested, and that the error is normal. Checking the assumptions of constant variance (homoscedasticity) and normality after fitting a linear regression model by exploring the model's residuals is a routine statistical practice. However, residuals explorations usually involve visual analyses of plots that are not easy to automate in computer platforms and, therefore, do not seem to be applicable in HTS campaigns fitting hundreds or thousands of regression models. Although statistical tests of homoscedasticity or normality are available and are amenable to automation, experienced statisticians know that these tests cannot be performed in isolation, that is, without additional residual analyses, because such tests can produce significant p-values in the presence of negligible deviations from the assumptions when the sample size is sufficiently large. However, it is a well known and fortunate fact that OLS estimators are fairly robust to deviations from the constant variance or normality assumptions, especially with sufficiently large samples [20,21]. Even more, under very general conditions, OLS estimators are consistent and asymptotically normal, even in the absence of homoscedasticity or in the presence of non-normal errors [20]. These conditions, along with some additional technical conditions that are satisfied by equation (5), also guarantee the asymptotic normality of $\varepsilon_{d}$ $[17,20]$, which is in the core of the statistical test that we used to examine the significance of $\varepsilon_{d}$. (The above technical conditions are stated in the mathematical result called "delta method" [20]). Therefore, the normality or homoscedasticity of the errors in model (3) is not a necessary condition for the applicability of our statistical test.

Nonetheless, if major deviations from standard OLS assumptions are suspected, the regression coefficients in model (3) can still be estimated through robust linear regression methods, which are implemented in some statistical packages. However, these methods usually have less statistical power than OLS when the general conditions for the asymptotic normality of OLS estimators are satisfied, which include many situations without homoscedasticity or normality. As a rule of thumb, we recommend that robust methods be used only when an unavoidable presence of many outliers across compounds is suspected. Also, it is a good practice to conduct a visual inspection of residuals for the models corresponding to those effective compounds that are selected for future experimental 
investigations, especially if their models show small $\mathrm{R}$ values. A visual inspection of the residuals of randomly selected models is also recommendable in order to identify potential estimation problems in the entire HTS campaign. In our study, residual analyses did not indicate evidence of serious departures from normality or homoscedasticity, except perhaps for the models corresponding to the OVCAR3 cell line. This is not totally unexpected because the OVCAR3 models had on average the lowest $R$ values (Table 4).

As another application, model (3) can be used to examine the significance of the effectiveness of a particular compound concentration. For each concentration $C_{i}$, the null hypothesis of non-toxicity $H_{0}: E_{d, i} \leq 0$ versus the alternative $H_{1}: E_{d, i}>0$ can be tested by using a 1-tailed t-test. Since $E_{d, i}>0$ if and only if $\lambda_{0}-\lambda_{d, i}>0$, the t-test statistic:

$$
t=\frac{\widehat{\lambda}_{0}-\widehat{\lambda}_{d, i}}{\operatorname{se}\left(\widehat{\lambda}_{0}-\widehat{\lambda}_{d, i}\right)}
$$

can be used, where $\lambda_{0} \hat{\text { and }} \lambda_{d, i}$ are the estimators of $\lambda_{0}$ and $\lambda_{d, i}$, respectively; and $\operatorname{se}\left(\lambda_{0} \hat{-}-\hat{\lambda_{d, i}}\right.$ is the standard error of $\hat{\lambda_{0}}-\hat{\lambda_{d, i}}$, obtained when the parameters in regression model (3) are estimated. We reject the null hypothesis $H_{0}$ when the p-value $P(T>t)$ is $<0.05$, where $T$ is at random variable with appropriate degrees of freedom. Interestingly, observe that, although $E_{d, i}$ is time dependent, the above statistical test is not because $t$ is a function of only the estimators of the growth rates $\lambda_{0}$ and $\lambda_{d, i}$. Provided that the plate wells have sufficient resources for the cells during the experiment, these growth rates should not be substantially affected by experiment durations.

\section{Acknowledgments}

Funded in part by NIH Grant (P20 RR015563/P30 RR030926; PI, Barbara Timmermann) (COBRE Program of the NCRR. KUMC Shared Resource).

\section{References}

1. Kalns JE, Millenbaugh NJ, Wientjes MG, Au JLS. Design and analysis of in vitro antitumor pharmacodynamic studies. Cancer Res. 1995; 55:5315-5322. [PubMed: 7585594]

2. Malich G, Markovic B, Winder C. The sensitivity and specificity of the MTS tetrazolium assay for detecting the in vitro cytotoxicity of 20 chemicals using human cell lines. Toxicology. 1997; 124:179-192. [PubMed: 9482120]

3. Freeman BC, Yamamoto KR. Disassembly of transcriptional regulatory complexes by molecular chaperones. Science. 2002; 296:2232-2235. [PubMed: 12077419]

4. Whitesell L, Lindquist SL. HSP90 and the chaperoning of cancer. Nat Rev Cancer. 2005; 5:761772. [PubMed: 16175177]

5. Holzbeierlein JM, Windsperger A, Vielhauer G. Hsp90: a drug target? Curr Oncol Rep. 2010; 12:95-101. [PubMed: 20425593]

6. Prodromou C. Strategies for Stalling Malignancy: Targeting Cancer's Addiction to Hsp90. Curr Top Med Chem. 2009; 9:1352-1368. [PubMed: 19860736]

7. Xu W, Neckers L. Targeting the Molecular Chaperone Heat Shock Protein 90 Provides a Multifaceted Effect on Diverse Cell Signaling Pathways of Cancer Cells. Clin Cancer Res. 2007; 13:1625-1629. [PubMed: 17363512]

8. Kim YS, Alarcon SV, Lee S, Lee MJ, Giaccone G, Neckers L, Trepel JB. Update on Hsp90 Inhibitors in Clinical Trial. Curr Top Med Chem. 2009; 9:1479-1492. [PubMed: 19860730]

9. Chaudhury S, Welch TR, Blagg BSJ. Hsp90 as a Target for Drug Development. ChemMedChem. 2006; 1:1331-1340. [PubMed: 17066389] 
10. Donnelly AC, Mays JR, Burlison JA, Nelson JT, Vielhauer G, Holzbeierlein J, Blagg BSJ. The design synthesis evaluation of coumarin ring derivatives of the novobiocin scaffold that exhibit antiproliferative activity. J Org Chem. 2008; 73:8901-8920. [PubMed: 18939877]

11. Shelton SN, Shawgo ME, Matthews SB, Lu Y, Donnelly AC, Szabla K, Tanol M, Vielhauer GA, Rajewski RA, Matts RL, Blagg BSJ, Robertson JD. KU135, a novel novobiocin-derived Cterminal inhibitor of the $90-\mathrm{kDa}$ heat shock protein, exerts potent antiproliferative effects in human leukemic cells. Mol Pharm. 2009; 76:1314-1322.

12. Burlison JA, Neckers LM, Smith A, Maxwell A, Blagg BSJ. Novobiocin: Redesigning DNA Gyrase Inhibitors for Selective Inhibition of Hsp90. J Am Chem Soc. 2006; 128:15529-15536. [PubMed: 17132020]

13. Yu XM, Shen G, Neckers L, Blake H, Holzbeierlein J, Cronk B, Blagg BSJ. Hsp90 Inhibitors Identified from a Library of Novobiocin Analogues. J Am Chem Soc. 2005; 127:12778-12779. [PubMed: 16159253]

14. Shonkwiler, RW.; Herod, J. Mathematical biology. An introduction with Maple and Matlab. 2. Springer; London: 2009.

15. Riss T, Moravec R, Beck M, Hannah R, Wilson K, Swanson R. A Viable Solution for Cytotoxicity Screening. Promega Notes. 2002; (8):2-5.

16. Stata Corp. Stata: Release 11 Statistical Software. StataCorp LP; College Station, TX: 2009.

17. Casella, G.; Berger, RL. Statistical Inference. 2. Duxbury; Pacific Grove, CA: 2002.

18. Benjamini Y, Yekutieli D. The control of the false discovery rate in multiple testing under dependency. Ann Stat. 2001; 29:1165-1188.

19. Hendricks P, Diaz FJ, Schmitt S, Sittampalam GS, Nirmalanandhan VS. Effects of Respiratory Mechanical Forces on the Pharmacological Response of Lung Cancer Cells to Chemotherapeutic Agents. Fundam Clin Pharm. 2011 [Epub ahead of print]. 10.1111/j.1472-8206.2011.00964.x

20. Shao, J. Mathematical Statistics. 2. Springer; New York: 2003.

21. Freedman, DA. Statistical Models. Theory and Practice. Cambridge University Press; New York: 2009. 


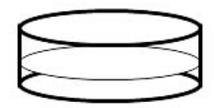

Cancerous or Normal Cell Line

Initial seeding of cell line based on cell growth curve

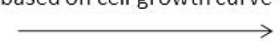

$\mathrm{H}_{\mathrm{o}}: \varepsilon_{\mathrm{d}} \leq 0$ (compound is non-toxic)

$\mathrm{H}_{1}: \varepsilon_{\mathrm{d}}>0$ (compound is toxic)

\section{$\varepsilon_{d}$ calculation to either accept} or reject the null hypothesis

Measure luminescence via PerkinElmer Envision or Tecan Safire

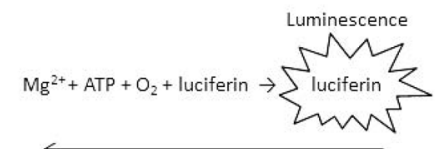

Cell lysis and luciferin oxidation catalized by luciferase 384-well plate for either $4,24,48$ or 72 hours

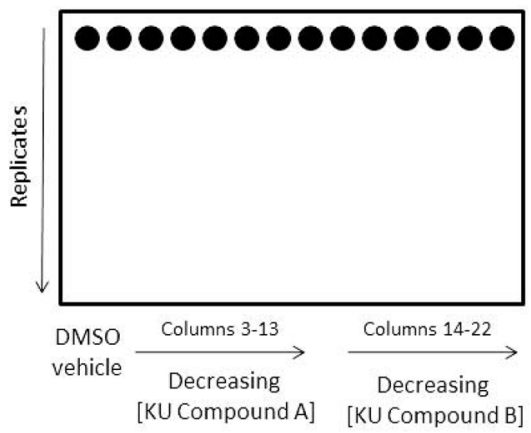

Incubation at $37^{\circ} \mathrm{C}$ and $5 \% \mathrm{CO}_{2}$

in a $95 \%$ humidified incubator

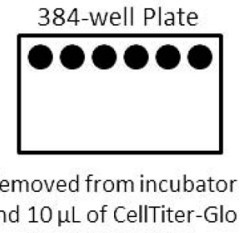

reagent is added

Fig. 1.

Schematic diagram of assay approach to determine $\varepsilon_{d}$ values for the 24 KU compounds, 17AAG and novobiocin. DMSO control was also tested. 


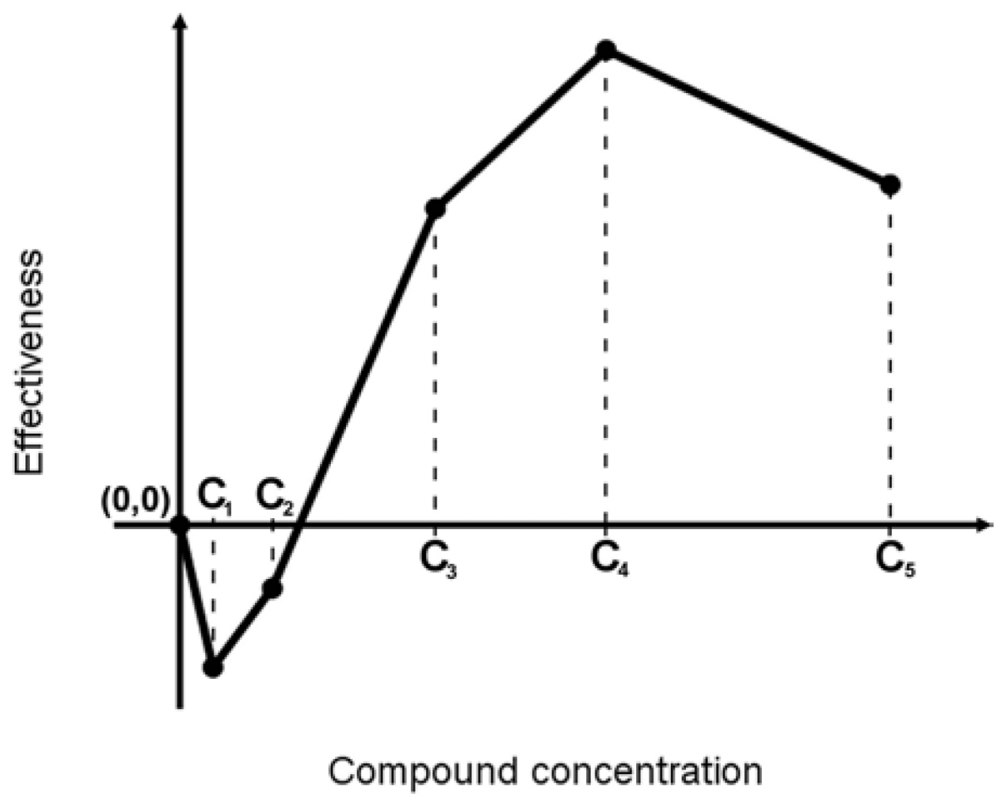

Fig. 2.

Schematic representation of the computation of the overall effectiveness $\varepsilon_{d}$ of a particular compound $d$, assuming that $n=5$ compound concentrations were used in HTS experiments. The overall effectiveness is the area under the curve of effectiveness $E_{d, i}$ versus compound concentration $C_{i}$, between a null concentration and the last concentration $C_{5}$, computed with the trapezoidal rule. Since $E_{d, i}$ may take negative values, $\varepsilon_{d}$ is the area of the polygon lying above the $\mathrm{x}$ axis minus the area of the polygon lying below. 


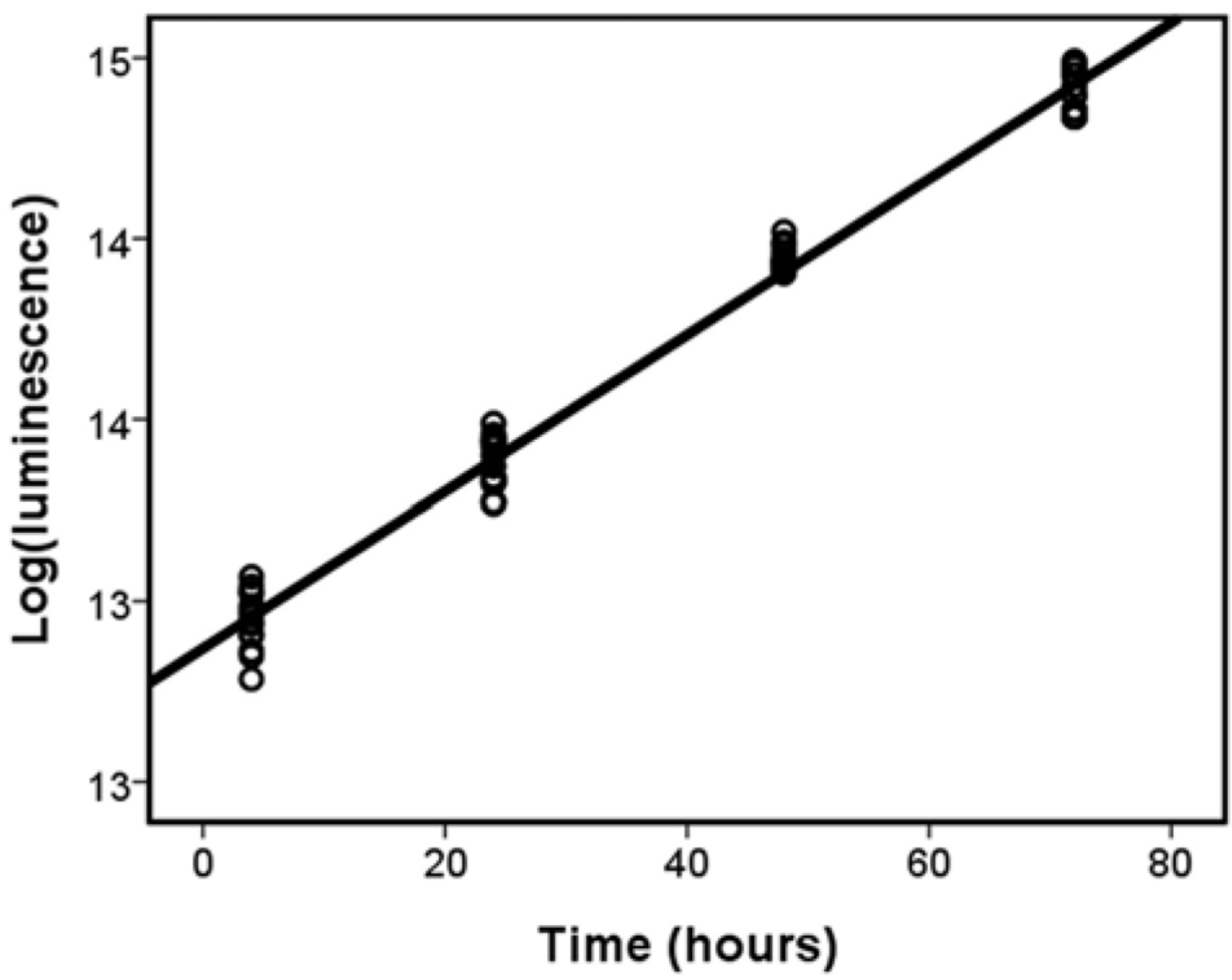

Fig. 3.

Plot of $\log$ (luminescence) versus exposure time for the MCF-7 cell line, using the observations from the cells who were exposed to DMSO vehicle alone $(\mathrm{N}=56)$. The close linear relationship suggests that the exponential growth model is a good representation of cell population growth under HTS conditions. Thirteen of the 19 investigated cell lines exhibited linear relationships between $\log$ (luminescence) and time, suggesting the adequacy of the exponential growth model, at least for these cell lines and exposure times examined. 
Table 1

Description of Investigated Cell Lines. All Lines were Cancerous, Except the Normal MRC-5

\begin{tabular}{|c|c|}
\hline Cell Line & Tissue Description \\
\hline A549 & Lung carcinoma, epithelial \\
\hline Coav-3 & Ovarian-adenocarcinoma, epithelial \\
\hline DRO & Thyroid-anaplastic \\
\hline HCC827 & Lung-adenocarcinoma, epithelial \\
\hline HCT-116 & Colorectal-carcinoma, epithelial \\
\hline HT-1376 & Urinary bladder carcinoma, grade 3 \\
\hline HT-29 & Colorectal-adenocarcinoma, epithelial \\
\hline JMAR & Oral cavity-squamous \\
\hline Jurkat, E6-1 & T lymphocyte-acute \\
\hline LNCaP & Prostate carcinoma \\
\hline MCF-7 & Breast-adenocarcinoma, epithelial \\
\hline MDA-1986 & Head and neck-squamous \\
\hline MRC-5 & Normal lung fibroblast \\
\hline NCI-H226 & Lung squamous cell carcinoma; mesothelioma \\
\hline NCI-H441 & Lung papillary adenocarcinoma \\
\hline NPA & Thyroid-papillary \\
\hline OVCAR3 & Ovarian-adenocarcinoma, epithelial \\
\hline PC3MM2 & Prostate-androgen independent \\
\hline SK-BR-3 & Breast-adenocarcinoma, epithelial \\
\hline
\end{tabular}




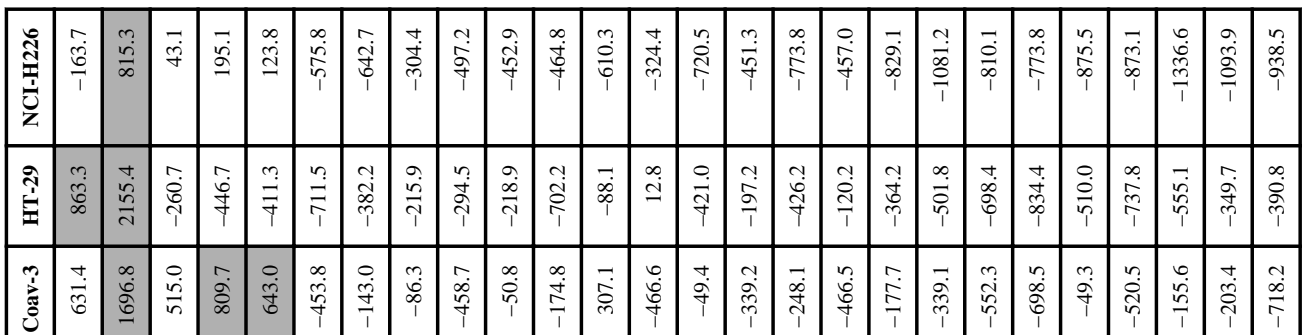

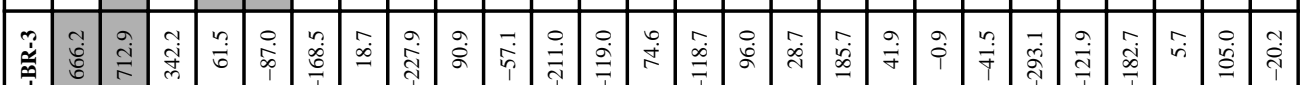

की

5

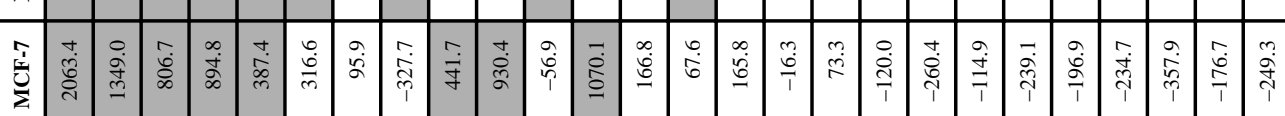

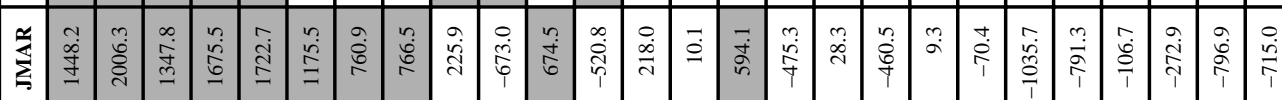

酸

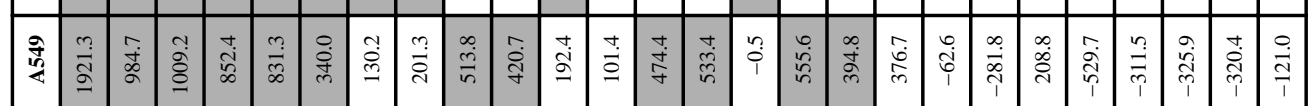

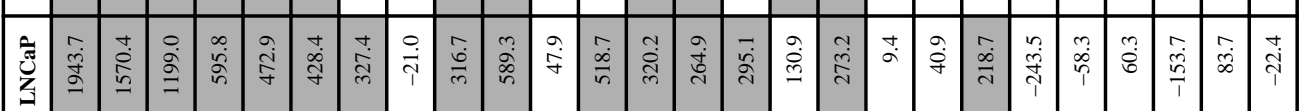

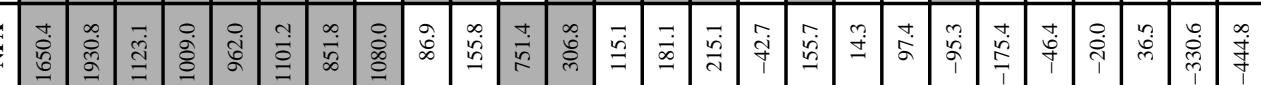

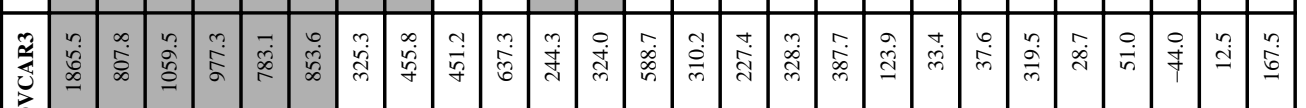

\%

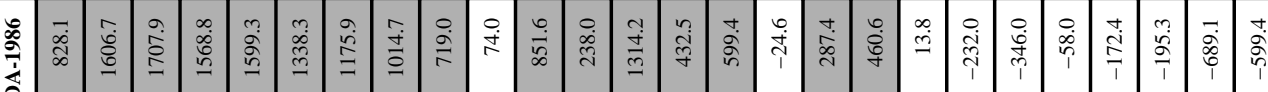

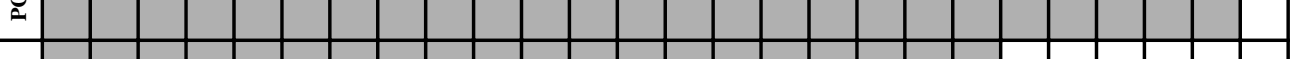

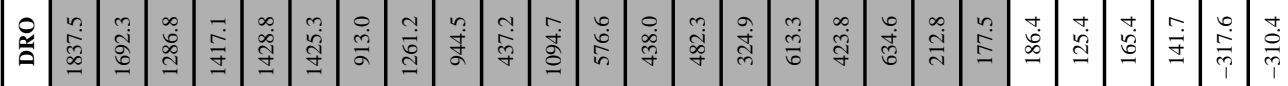

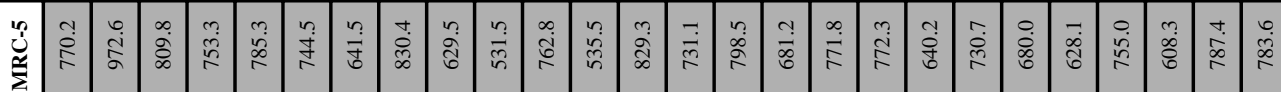

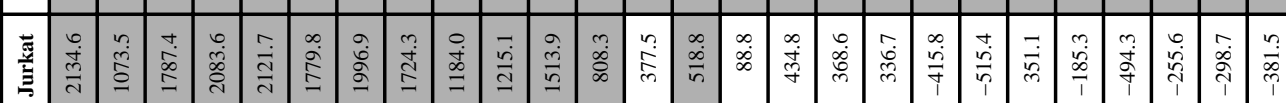

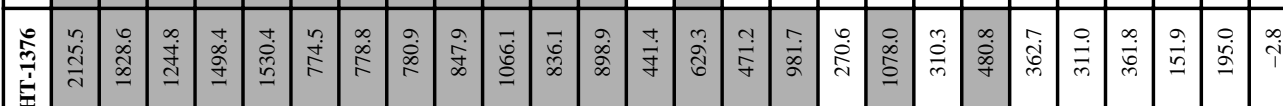

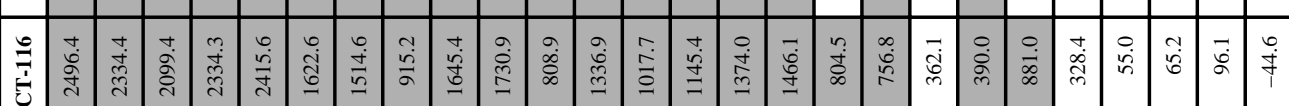
约

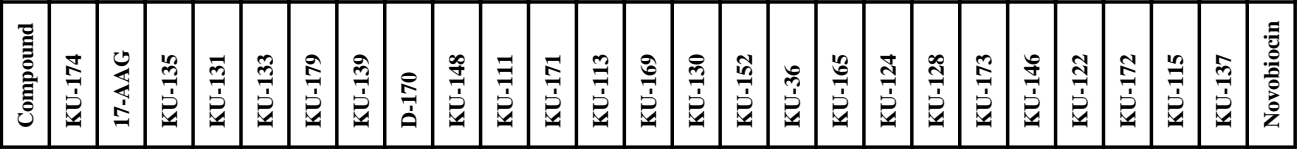




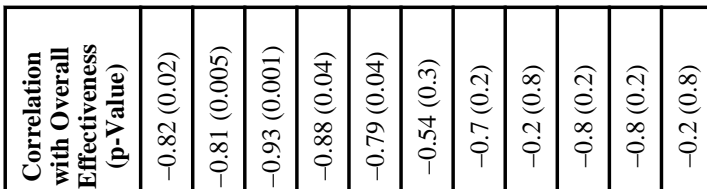

$\hat{e}$
$\hat{e}$
$n$
$i$
$i$

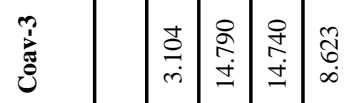



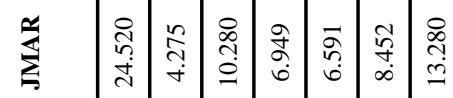

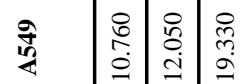

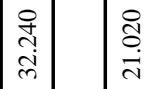

高

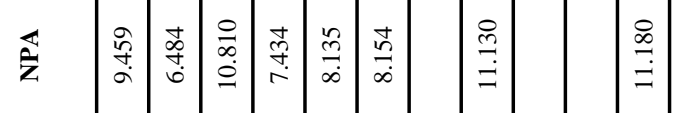

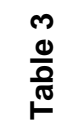

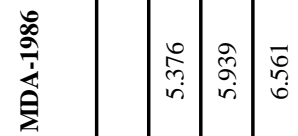

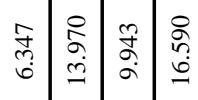

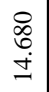

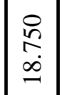

$\stackrel{\substack{3 \\ \frac{1}{2}}}{\frac{1}{2}}$

웅

$\stackrel{00}{:}$

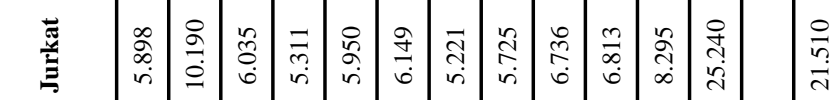

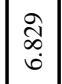

$\stackrel{\substack{2 \\:}}{=}$

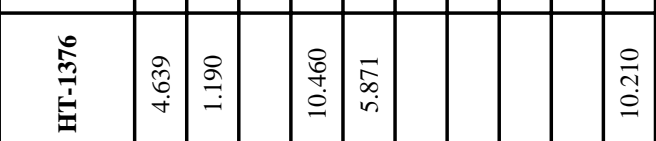

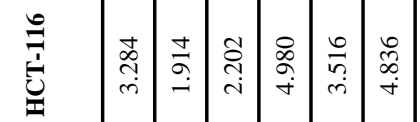

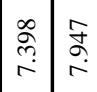
ํㅗㄱ
号
辛

\begin{tabular}{ll|l|l|l|l|l|l|l|l|l|l|l|l|l|l|l|l}
0 & 0 \\
\hline
\end{tabular} 


\begin{tabular}{|c|c|}
\hline \multicolumn{2}{|c|}{ Diaz et al. } \\
\hline 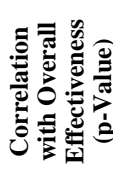 & \\
\hline 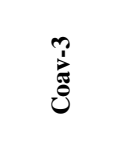 & $\begin{array}{l}\widehat{\delta} \\
\stackrel{0}{0} \\
0 \\
\infty \\
i\end{array}$ \\
\hline 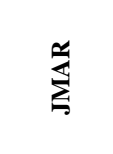 & $\begin{array}{l}\frac{\sigma}{0} \\
\stackrel{0}{0} \\
\\
\hat{i}\end{array}$ \\
\hline 密 & $\begin{array}{l}\widehat{\delta} \\
\stackrel{0}{0} \\
\stackrel{0}{\infty} \\
i\end{array}$ \\
\hline$\widehat{\hat{z}}$ & $\begin{array}{l}\text { f. } \\
\stackrel{0}{0} \\
0 \\
e \\
0 \\
i\end{array}$ \\
\hline$\frac{\mathscr{0}}{\stackrel{\circ}{\dot{a}}}$ & $\begin{array}{l}\widehat{\sigma} \\
\dot{0} \\
\dot{0} \\
\underline{a} \\
o \\
i\end{array}$ \\
\hline$\stackrel{\text { ॥ }}{\circ}$ & $\begin{array}{l}\widehat{\bar{\theta}} \\
\dot{0} \\
\stackrel{\dot{0}}{\sigma} \\
\stackrel{0}{i}\end{array}$ \\
\hline 总 & \\
\hline 莺 & $\begin{array}{l}\widehat{\sigma} \\
\stackrel{0}{0} \\
\stackrel{0}{0} \\
\infty \\
0 \\
i\end{array}$ \\
\hline 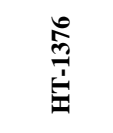 & $\begin{array}{l}\widehat{0} \\
\stackrel{0}{\infty} \\
\stackrel{i}{i}\end{array}$ \\
\hline 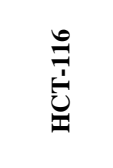 & $\begin{array}{l}\widehat{\widehat{a}} \\
\stackrel{0}{0} \\
\stackrel{0}{0} \\
\infty \\
i \\
i\end{array}$ \\
\hline 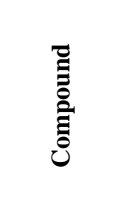 & 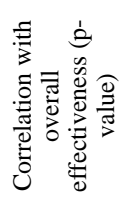 \\
\hline
\end{tabular}

Comb Chem High Throughput Screen. Author manuscript; available in PMC 2013 May 16. 
Table 4

Summary of Correlation Coefficients R Between Observed and Predicted Log-Transformed Luminescence Measures, where the Predictions were Computed with the Proposed Linear Regression Model. The Relatively High Correlations Described in the Table Suggest that the Model Fit Dose-Response Data from HTS Toxicity Screens Remarkably Well. ${ }^{a, b}$

\begin{tabular}{|c|c|c|c|}
\hline Cell line & Minimum of 26 Correlations & Maximum of 26 Correlations & Average of 26 Correlations \\
\hline HCT-116 & 0.94 & 0.98 & 0.97 \\
\hline HT-1376 $^{c}$ & 0.84 & 0.91 & 0.88 \\
\hline Jurkat $^{d}$ & 0.86 & 0.92 & 0.90 \\
\hline MRC-5 $^{e}$ & 0.59 & 0.83 & 0.65 \\
\hline DRO & 0.86 & 0.98 & 0.97 \\
\hline PC3MM2 & 0.88 & 0.95 & 0.94 \\
\hline HCC827 & 0.77 & 0.95 & 0.93 \\
\hline MDA-1986 & 0.90 & 0.98 & 0.97 \\
\hline OVCAR3 & 0.66 & 0.76 & 0.69 \\
\hline NPA & 0.91 & 0.95 & 0.93 \\
\hline LNCaP & 0.93 & 0.99 & 0.99 \\
\hline A549 & 0.94 & 0.98 & 0.97 \\
\hline JMAR & 0.91 & 0.97 & 0.95 \\
\hline MCF-7 & 0.93 & 0.96 & 0.95 \\
\hline NCI-H441 & 0.68 & 0.83 & 0.76 \\
\hline SK-BR-3 $f$ & 0.82 & 0.91 & 0.86 \\
\hline Coav-3 & 0.53 & 0.85 & 0.60 \\
\hline HT-29 & 0.98 & 0.99 & 0.99 \\
\hline NCI-H226 & 0.97 & 0.93 \\
\hline
\end{tabular}

${ }^{a}$ All correlations that are summarized in the table were significant. Each correlation corresponded to one investigated compound.

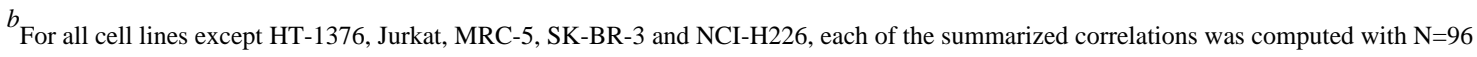
luminescence measures; 56 of these measures corresponded to DMSO alone, and the remaining measures to compound concentrations greater than 0 . For the other cell lines, one time point from DMSO-alone data was excluded from computations and, therefore, 82 luminescence measures were used to compute each correlation.

${ }^{c}$ Luminescence measures obtained after 4 hours under DMSO alone were excluded from computations (see explanation in Model Fit Section).

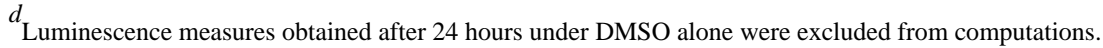

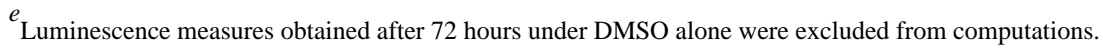

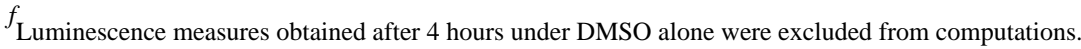

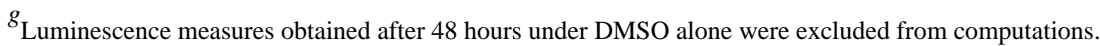

